\title{
Molecular characterization of dasheen mosaic virus isolates infecting edible aroids in India
}

\author{
B. BABU, V. HEGDE
}

Division of Crop Protection, Central Tuber Crops Research Institute, Thiruvananthapuram Kerala - 695017, India

Received August 13, 2013; accepted February 19, 2014

\begin{abstract}
Summary. - Dasheen mosaic virus (DsMV) infecting three major edible aroids namely Amorphophallus paeoniifolius, Colocasia esculenta, and Xanthosoma sagittifolium cultivated in India was characterized. Infected plants showing typical DsMV symptoms were subjected to reverse transcription-polymerase chain reaction, and an amplification of a $963 \mathrm{bp}$ fragment which encoded the coat protein (CP) gene was obtained. BLAST analysis of the cloned DNA amplicon revealed the identity of the virus to be that of DsMV. Sequence identity matrix of the nucleotide sequences among the three isolates showed that the DsMV isolate infecting A. paeoniifolius and C. esculenta shared an identity as high as $93 \%$, while the DsMV isolate from X. sagittifolium shared an identity of only $73 \%$ and $76 \%$ with the DsMV isolates from A. paeoniifolius and C. esculenta, respectively. Comparative analysis of the coat protein of the three DsMV isolates showed the presence of DVG motif (A. paeoniifolius and C. esculenta) and DTG motif in X. sagittifolium and several varying potential threonine and asparagine rich $\mathrm{N}$-glycosylation motifs. Single amino acid substitution of the several conserved motifs occurs in all the three DsMV isolates. This is the first characterization of DsMV isolates infecting A. paeoniifolius, C. esculenta, and $X$. sagittifolium plants in India.
\end{abstract}

Keywords: Amorphophallus paeoniifolius; coat protein; Colocasia esculenta; potyvirus; RT-PCR; Xanthosoma sagittifolium

\section{Introduction}

Tuber crops form the means of sustenance for millions of people in the tropical and subtropical world. They have a higher biological efficiency as food producers and show the highest rate of dry matter production per day per unit area among all the crops. These crops are known to supply cheap source of energy especially for the weaker sections of the population. These attributes make tuber crops ideal for cultivation in the less developed countries of the world.

In India, among the tuber crops of economic importance, edible aroids of the family Araceae, including Amorphophallus paeoniifolius (Elephant foot yam), Colocasia esculenta (Taro) and Xanthosoma sagittifolium (Arrowleaf elephant ear,

E-mail: binoybabu@ufl.edu; phone: +1-850-570-6686.

Abbreviations: DsMV = dasheen mosaic virus; VMV = vanilla mosaic virus; $\mathrm{RDP}=$ Recombination detection programme; $\mathrm{CP}=$ capsid protein; $\mathrm{Nib}=$ gene for RNA-dependent RNA polymerase
Cocoyam, Tannia) plays a very important role in the socioeconomic development of the country. Elephant foot yam is widely used as vegetable in various cuisines and in preparation of indigenous ayurvedic medicines (Misra et al., 2002). It has become a cash crop and is gaining popularity due to its high production potential (50-60 t/ha), nutritional and medicinal values, and high economic returns (Srinivas and Ramanathan, 2005). Taro is grown primarily as a vegetable food for its edible corms and forms an important staple in the diets of people throughout the tropics. In addition, taro is known to have medicinal properties, and has a very valuable place in the world agriculture scenario as this crop has an important role in the subsistence economies and in crop diversification. Another important edible tropical tuber crop, Cocoyam (Jennings, 1987; Onwueme and Charles, 1994), grown for its starchy corms forms an important source of nutrition for both humans as well as animals (Ndoumou et al., 1995; Tambong et al., 1997; Nyochembeng and Garton, 1998). 
All three important edible aroids cultivated in India are vegetatively propagated and are affected by viral disease. In spite of their importance in the diets of poor farmers, very little attention has been paid to study the diseases occurring on these crops in India. Among the field diseases of these edible aroids, infection caused by DsMV of the genus Potyvirus is widely spread. Repeated use of virus infected planting materials has increased the disease incidence in these edible aroids in the recent years. In the case of Elephant foot yam, occurrences of mosaic, puckering, leaf distortion, filiformy or shoestringing type symptoms are common. In India, Pandit et al. (2001), first reported the association of a DsMV with the mosaic disease of Elephant foot yam based on serological studies from the leaf samples collected from West Bengal. The infection caused by DsMV on the plant appeared to be severe resulting in reduced tuber yields. Nehalkhan et al. (2006), reported a $24-88 \%$ mosaic incidence with yield losses of 3.5-38\% in Elephant foot yam growing fields, from the state of Uttar Pradesh. Disease incidence of 5-10\% was observed in other major Elephant foot yam growing states of India (Kerala, Andhra Pradesh, and Orissa) (Binoy et al., unpublished data).

In case of taro, the leaves show a mosaic pattern along the veins, causing variation patterns in color, shapes, and sizes of the leaf, and sometimes produce whitish feathery symptoms, and reduced corm yield. Occurrence of mosaic disease on taro has been reported from Thiruvananthapuram, with typical symptoms resembling that of infection with DsMV (Malathi and Shanta, 1981). In case of Cocoyam, also whitish feathery mosaic symptoms are commonly observed. The other visible symptoms on the plants include leaf distortion, vein chlorosis, mosaic feathering along the veins (Zettler et al., 1989), and in case of a severe attack, stunted plants result in reduced tuber yield. Our preliminary studies with immunosorbent electron microscopy and RT-PCR using potyvirus group specific primers have showed the association of DsMV with all the three infected edible aroids cultivated in India (Binoy et al., 2010).

DsMV, an aphid-transmitted virus, was found to infect a wide variety of cultivated aroids and ornamental plants worldwide (Zettler et al., 1978; Zettler and Hartman, 1986, 1987; Brunt et al., 1996). Partial sequence characterization of DsMV infecting Colocasia (Pappu et al.,1994), Xanthosoma (Reyes et al., 2009) and Zantedeschia (Chen et al., 2001) and complete genome sequence from Zantedeschia have been reported (Chen et al., 2001). However no sequence information of DsMV from A. paeoniifolius, C. esculenta, and X. sagittifolium in India has so far been reported. Therefore, the objective of the present study was to characterize the sequences of the CP gene of three DsMV isolates infecting these crops and to analyze the molecular and phylogenetic relationship among these isolates.

\section{Materials and Methods}

Virus source and RNA extraction. Diseased A. paeoniifolius, C. esculenta, and X. sagittifolium plants showing foliar mosaic symptoms were collected from Central Tuber Crops Research Institute (Trivandrum, Kerala) field, and total RNA was extracted from $100 \mathrm{mg}$ of infected leaves using the Qiagen RNeasy Plant mini kit (Qiagen, USA), according to the manufacturer's protocol.

$R T-P C R$. Total RNA was subjected to RT-PCR using a one step RT-PCR kit (Finnzymes) in a reaction mixture $(20 \mu \mathrm{l})$ containing $5 \mu \mathrm{l}$ RA, $1 \mu \mathrm{l}$ of $5 \mu \mathrm{mol} / \mathrm{l}$ oligo $(\mathrm{dT})_{16}, 1 \mu \mathrm{l}$ of $5 \mu \mathrm{mol} / \mathrm{l}$ each of $\operatorname{DsMV}(\mathrm{I})-\mathrm{F}$ and DsMV(I)-R primers, $2.0 \mu \mathrm{l}$ of $10 \mathrm{x} / 5 \mathrm{x}$ reaction buffer, $0.5 \mu \mathrm{l}$ of $10 \mathrm{mmol} / \mathrm{l}$ dNTPs, $0.5 \mu \mathrm{l}$ of $50 \mathrm{mmol} / 1 \mathrm{MgCl}_{2}, 0.25$ $\mu \mathrm{l}$ of AMV reverse transcriptase and $1.0 \mu \mathrm{l}$ of Dynazyme DNA Polymerase in Eppendorf Mastercycler Gradient ES with the following profile: $48^{\circ} \mathrm{C}$ for $45 \mathrm{~min}, 94^{\circ} \mathrm{C}$ for $2 \mathrm{~min}$, followed by 35 cycles of $94^{\circ} \mathrm{C}$ for $30 \mathrm{sec}, 58^{\circ} \mathrm{C}$ for $1 \mathrm{~min}, 72^{\circ} \mathrm{C}$ for $1 \mathrm{~min}$ and one cycle of $72^{\circ} \mathrm{C}$ for $10 \mathrm{~min}$. Degenerate forward primer DsMV (I)F and specific reverse primer DsMV (I)R were designed 21 nucleotides upstream of the 5 ' end of the CP gene comprising the partial NIb region (DsMV (I)F 5'-GATGCCTTTRMCTTTGARNTGWKSKGTG-3') and in the 3 'untranslated region downstream of the CP gene (DsMV (I) R 5'-GCCAATAACTGTGGCCTGTT-3'), respectively, using the Primer Premier Software version 5, using the DsMV sequences available in the GenBank. The amplified product was analyzed on $1 \%$ agarose gel, stained with ethidium bromide and photographed with a UV-gel doc system (Alpha Imager HP, California).

Cloning and sequencing. The amplified PCR products were purified using the Gel extraction kit (Qiagen), cloned into the pGEM-T Easy vector (Promega) and transferred into Escherichia coli strain DH5a (Sambrook and Russel, 2001). Ten positive clones of each isolate carrying the insert were sequenced using T7 and SP6 primers and also with primers DsMV (I)F and DsMV (I)R.

Phylogenetic analysis. Sequences were edited using the BIOEDIT Software (Hall, 1999). Nucleotide and the deduced amino acid sequences were compared with those in the NCBI database using the Basic Local Alignment Search Tool (BLAST; http://www.ncbi.nlm. nih.gov/blast/), with Blastn and Blastp, respectively. Deduced amino acid sequences were analyzed using the GCG program MOTIFS (Devereux et al., 1984). Multiple sequence alignment of the virus isolates and the identity percentage between aligned sequences were calculated using Clustal W (Thompson et al., 1994). Sequence identity matrix of the DsMV isolates under study was done using the BIOEDIT software. Phylogenetic analysis using nucleotide sequences was done between DsMV and other selected members of the genus Potyvirus. From the aligned sequences, a phylogenetic tree was constructed using the neighbor-joining method (Tajima and Nei, 1984) with 100 bootstrap replicates, and the tree was constructed using the TREECON software (Van de Peer and De Wachter, 1994).

Recombinant analysis. Recombination analysis of the three isolates was carried out in order to determine the genetic relation- 
(a)

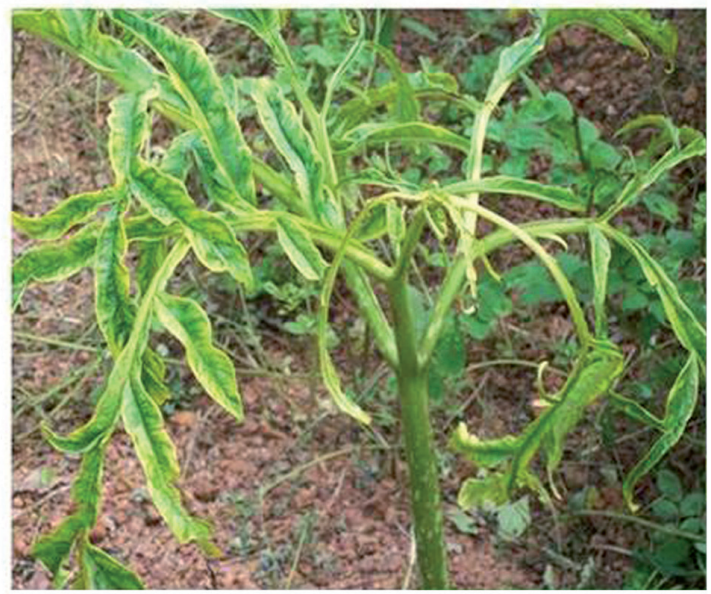

(b)

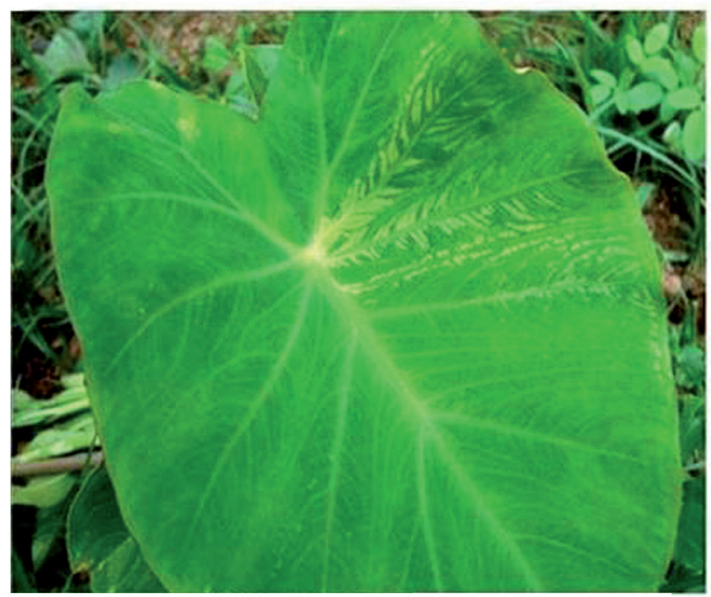

(c)

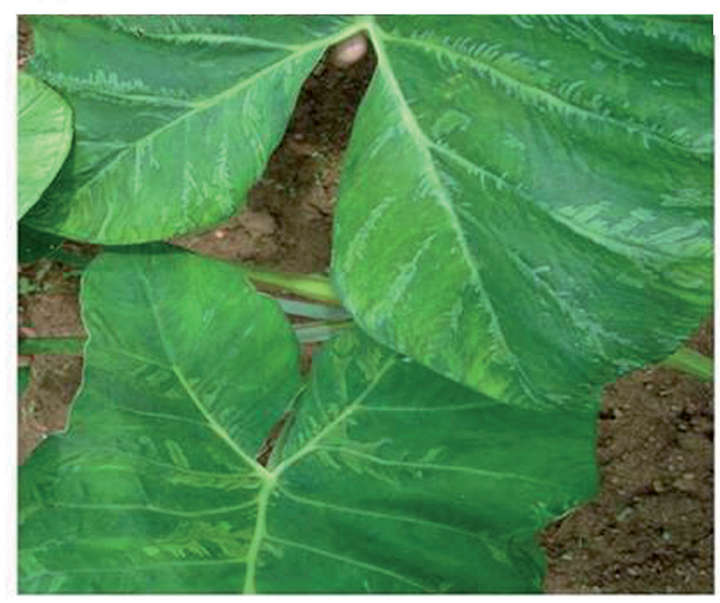

Fig. 1

Symptoms on leaves of edible aroids infected by DsMV (a) Amorphophallus paeoniifolius, (b) Colocasia esculenta, (c) Xanthosoma sagittifolium. ship, using different methods of the Recombination detection programme (RDP Version 3.0) (Martin and Rybicki, 2004).

Sequences. Nucleotides sequences have been submitted to NCBI database under the accession number: DsMV-Amp1 (FJ160764), DsMV-Ta1 (HQ207541) and DsMV-Xan1 (HQ207542). In case of DsMV-Amp1 (FJ160764), the sequence submitted was from an earlier clone (1223 bp) containing the NIb, CP, and the 3'UTR region, from which specific primers were designed for the amplification in the present study.

\section{Results and Discussion}

RT-PCR analysis of total RNA from infected leaves of all three aroid species (Fig. 1) with DsMV specific primers resulted in 963 bp amplicon (Fig. 2), which potentially encoded the complete CP gene of 313 amino acids. Sequence analysis of the three isolates using BLAST indicated that the virus is DsMV. All the three isolates shared $80-90 \%$ nt identity with the other DsMV sequences available in the GenBank. BLAST analysis of the CP nucleotide sequence indicated that the three DsMV isolates also showed a higher identity at the nucleotide and amino acid level respectively, with that of vanilla mosaic virus (VMV) (86-88\% nt and 94-95\% aa), and an moderately higher identity with that of Bean common mosaic virus (BCMV) (78-80\% nt and $82-85 \%$ aa) (data not shown). The identities between these viruses and the dendrogram clustering of these viruses together (Fig. 3) clearly indicates the close relationship among these viruses, as was also reported earlier (Adams et al., 2005; Reyes et al., 2009; Farreyrol et al., 2006). DsMV isolates from the three edible aroids under study differed from two other DsMV isolates (AY994104, AY994105) and with one VMV (AJ616719) from GenBank, in lacking 36 nucleotides at the 5'-end region of the CP and 54 nucleotides (140-194) compared to 9 DsMV isolates (AM910398, DMU00122, AF169832, EF199550, DQ925464, AM910407, AM910401, AM994104, and AY994105) and one VMV isolate (AJ616719) (data not shown).

Comparative analysis of the nucleotide sequence identity matrix of the three isolates indicated that DsMV-Amp1 and DsMV-Ta1 shared a higher identity ratio of 0.927 , while DsMV-Xan1 showed an identity ratio of 0.756 and 0.728 with DsMV-Amp1 and DsMV-Ta1 respectively (Table 1). These data clearly show that the DsMV isolates obtained from A. paeoniifolius and C. esculenta could probably be the similar isolates, while the DsMV isolate from Xanthosoma $s p p$. could be a different strain. Comparative to other DsMV isolates from the GenBank, the identity matrix shows that the DsMV-Amp1 and DsMV-Ta1 share the highest identity among themselves, while the DsMV-Xan1 showed the highest identity (0.8) with that of DsMV isolate from China (Acc. 
No: EU420058), followed by the highest identity with that of DsMV-Amp1(0.76). The lower identity of the DsMVXan1 with other viral isolates clearly proves that the isolate is a completely new strain of DsMV. Moreover since the isolates were collected from the same geographical location, the data clearly prove the presence of varying DsMV strains/ isolates in the same location. Moreover in our other studies we have analyzed 12 different DsMV isolates (HQ207529 to HQ207540) from A. paeoniifolius collected from different geographical locations of Kerala, which exhibited high variability among the isolates (Binoy et al., unpublished data). Analysis of our viral isolate DsMV-Amp1 with these viral isolates from the same host indicated a higher identity ranging between $74-96 \%$, while the DsMV isolate from the host C. esculenta-DsMV-Ta1, indicated a lower identity with these isolates ranging from $64-71 \%$ (data not shown). The DsMV isolate from Xanthosoma - DsMV-Xan1 indicated an identity ranging between $72-86 \%$ with the 12 different DsMV isolates from A. paeoniifolius. These data prove that the DsMV isolates from the same host shared a higher identity, while from the different host appeared to be variable, except in the case of DsMV-Xan1 which showed an identity of $84-86 \%$ with certain DsMV isolates. This proves that the Xanthosoma isolate, percentage of identity is somewhere between the divergent line and that of the DsMV isolates from A. paeoniifolius. Moreover the DsMV isolate from the host plant C. esculenta (DsMV-Ta1) showed the highest identity with that of the DsMV-Amp1 isolate from a different host plant (from Trivandrum), and a lower identity with that of the other DsMV isolates from A. paeoniifolius from different locations. The constructed phylogenetic tree clearly shows that the two isolates DsMV-Amp1 and DsMV-Ta1 share a higher identity and are grouped in similar clade, while DsMV-Xan1 forms an entirely different clade (Fig. 3). The similar geographical location of the three DsMV isolates and the higher and lower sequence identity among these isolates could be attributed to the occurrence of recombination events in the evolution of these isolates. Recombination analysis of the three viral isolates using RDP showed the presence of single recombination event in the coat protein region of the viral isolates (Fig. 4). The recombination events were detected by GENECONV, BootScan, MaxChi, Chimaera, SiScan and 3Seq programmes of the RDP, with a recombination frequencies ranging from $1.281 \times 10^{-02}$ to $7.181 \times 10^{-01}$ (Bonferroni P-value 0.05). The recombination analysis indicated the possibility of DsMV-Amp1 to be a recombinant of DsMV-Xan1 (major parent) and DsMVTa1 (minor parent). However recombinant analysis of the other regions of the genome, which could possibly provide an insight in to the occurrence of these isolates with varying identities in the same geographical locations, needs to be carried out. The possibilities of recombination events are high in case of viral isolates found in similar geographical locations

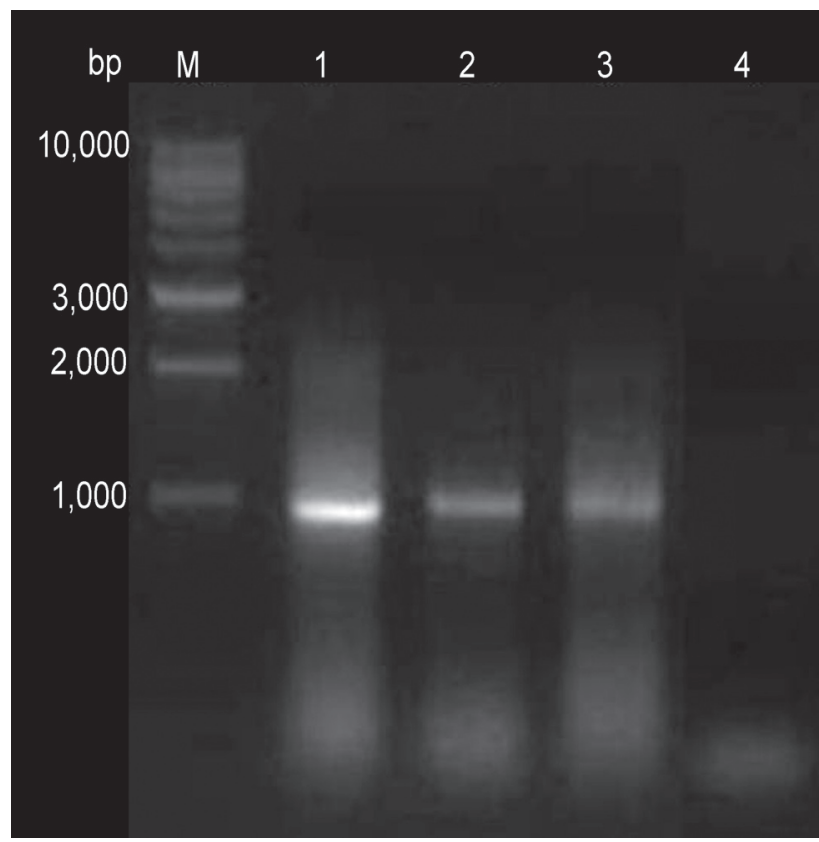

Fig. 2

RT-PCR analysis of DsMV infected edible aroids

Lane M: $1 \mathrm{~Kb}$ marker; lane 1: infected A. paeoniifolius sample; lane 2: infected $C$. esculenta samples; lane 3: infected X. sagittifolium sample; lane 4: negative control.

(Bateson et al., 2002; Garcia-Arenal et al., 2001), and could be adopted for the better adaptability to the environment and to the suitable host. Moreover, the other possible causes for the genetic variation such as genetic drift and selection (Garcia-Arenal et al., 2001) also need to be analyzed for a much better understanding.

Multiple sequence alignment of the $\mathrm{CP}$ of the three viral isolates under study showed that the $\mathrm{N}$-terminal region of the $\mathrm{CP}$ varies considerably; however, two thirds of the C-terminal were highly conserved. Protease cleavage sites in potyvirus polyprotein sequences for the production of the $\mathrm{CP}$ are $\mathrm{Q} / \mathrm{A}$, $\mathrm{Q} / \mathrm{S}$ or Q/G residues (Pappu et al., 1994). DsMV-Amp1, DsMVTa1 and DsMV- Xan1 have the potential protease cleavage site at position 7 and 8, between Q/A to produce a CP of 313 amino acids (Fig. 5). The presence of the sequence DEVVL in the NIb region, upstream of the glutamine residue of the Q/A site at position 6 suggests that this is the protease digestion site in almost all the isolates. This region also correlates with the protease sites for other potyviruses (Yu et al., 1989). The presence of the sequence ADDTV following the first putative cleavage site indicates that this site is likely active, as reported for Tobacco etch virus and Tobacco vein mottling virus (Pappu et al., 1994; Yu et al., 1989). In DsMV-Amp1 and DsMV-Xan1, the site ADDTV is conserved, while in DsMV-Ta1, a single amino acid substitution of V/G occurs. A DVG motif (residues 6 to 8 ) was observed in the DsMV-Amp1 and DsMV-Ta1, and 


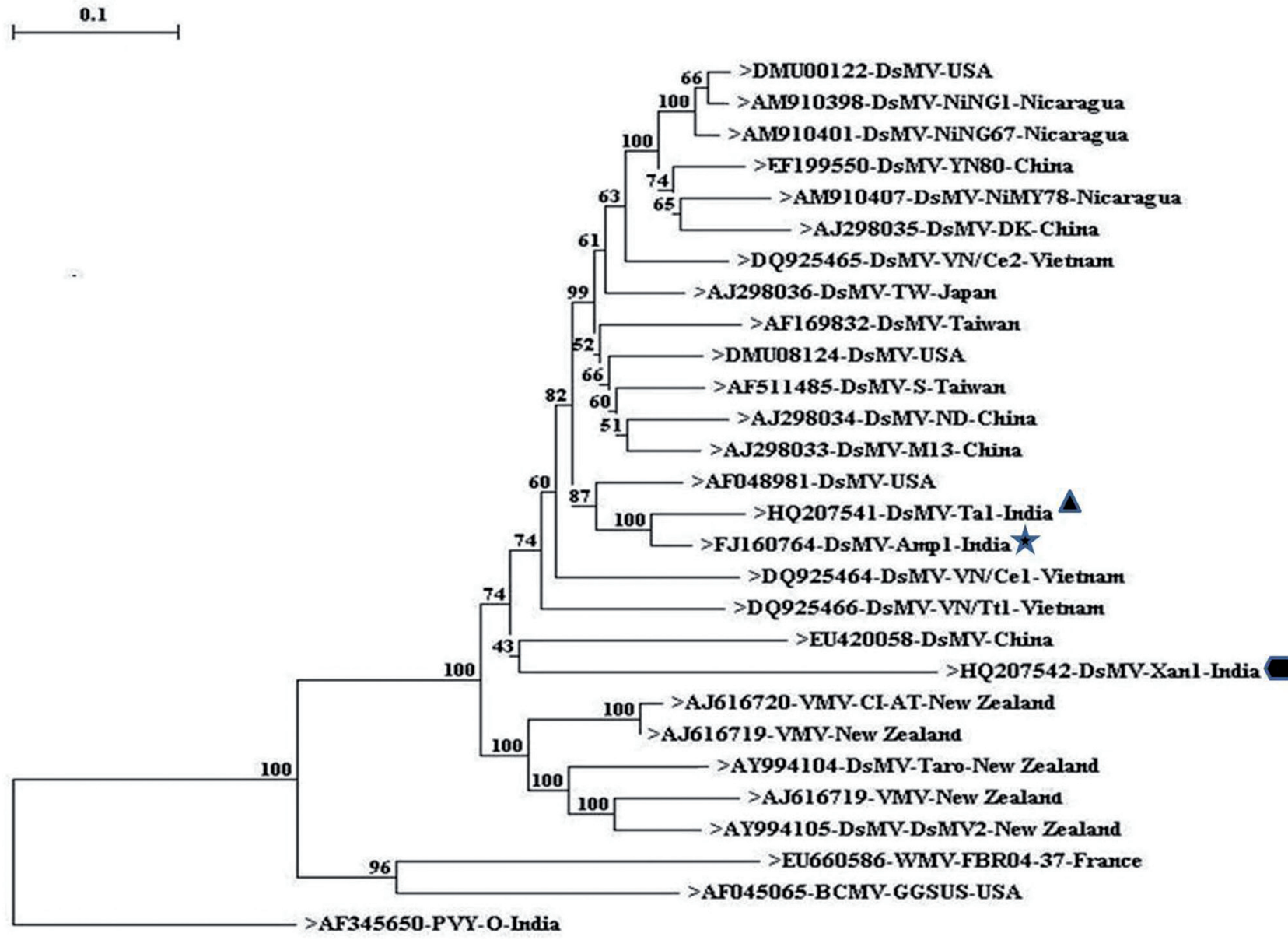

Fig. 3

Phylogenetic tree illustrating relationships of the three DsMV isolates DsMV-Amp1 $\star$, DsMV-Ta1 $\Delta$ and DsMV-Xan1 $\square$ with different viral isolates using neighbor-joining method with 100 bootstrap replicates and with potato virus $\mathrm{Y}$ as outgroup

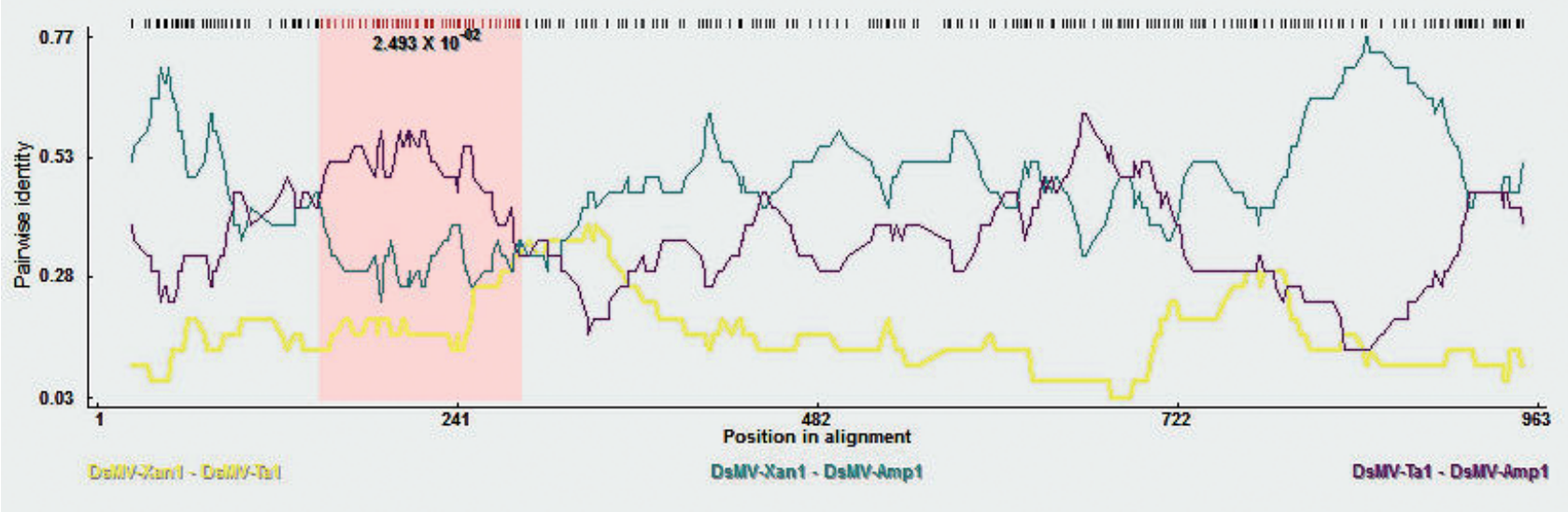

Fig. 4

Recombination rate plot of the three DsMV isolates using Recombination detection programme 
FJ160764-DsMV-Amp1 HQ207541-DsMV-Ta1 HQ207542-DsMV-Xan1

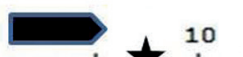

20

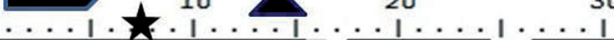
30 $\overbrace{50}^{40 \ldots \ldots}{ }_{60}$ HDEVVLCADDTVDVGKIINTTKVTETKTEAAGGGNNTNNTTPEVNNTINDNPPPPPEAAP HDEVVLC્ADDTGDVGC्रATLIAKVTCAKTEAAGGGNNTNSTTPEADNTTSNNPPPPPEAAP HDEVVLÇADDTVDTGPFINTT'TIGRETIATOGGNNTNNTTESVEGTTSEQTPPPPFRిVP
80 90 $\longrightarrow$

110

120 KATETEANKCVVPSTNEKGKEVVKDVNAGTSGTYSVPRINRITHKNNLPIVKGKCIININ KATETEANKCQVVPSTNEKGKEI IKDVNAGTSGTYAVPRLDRITENNNLPLFKGKCIININ KVLINEVAKSVDPSVKEKERKVVRDVN VGTSGVFSVPRINRITKKNNLPIVKGRCIININ HQ207542-DsMV-Xan1 140 150 160 $\ldots|\ldots| \ldots|\ldots| \ldots|\ldots| \ldots|\ldots| \ldots|\ldots| \ldots|\ldots| \ldots|\ldots| \ldots|\ldots| \ldots \mid$ HLIEYKPEQRD IF NTRATHTQFEVWYNAVKREYELDDECMMHIVNNGFNVWCIDNGTSPDI HLIEYK PEQRDIFNTRATHTQFEFWYNAVKREYELEDECMHHINNGFNVWCIDNGTSPDI HQ207541-DsMV-Ta1 HIMEYKRKQRDIINSRSTHTQFDIWYNAVKREYELQDDHMHIVNNGFNVWCIYNGTSPDI HQ207542-DsMV-Xan1

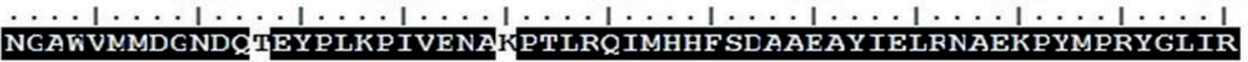
HQ207541-DSMV-Ta1 NGAKVMMDGNDÇVEYPLKPIVEH:ÅPYPTSRQIMHHF SDAAEAYIELRNAEKPYMPRYGLIR HQ207542-DsMV-Xan1

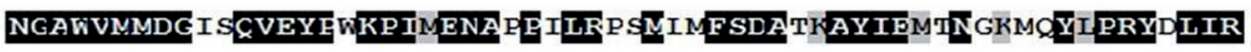
250 260 270 280 290 300 FJ160764-DsMV-Amp1 HQ207541-DSMV-Ta1 NLRDASIARYAFDFYEVNSKTEVRAREAVACMKAAALSNVTTRLFGLDGNVSTSSENTER NLRDASI ARYAFDFYEVNSKTFVRAREAVACMM AAALSNVTTRLFGLDGNVSTSSEN $\mathrm{PER}$ HQ207542-DsMV-Xan1 NLFEATI ARYAFDFYEVNSKTEIRAREAVACMMKAAALSNVTTRLFGLDGNVATIASENTER

310 320

FJ160764-DsMV-Amp1 HTAKDVTENMHTLLGVSSPQ * HQ207541-DsMV-Ta1 HTAKDVTEYMHTLLGVSSPQ * HQ207542-DsMV-Xan1 HTERNRPQNNCATGMVSSPQ *

\section{Fig. 5}

Deduced amino acid sequence of the coat protein of DsMV of A. paeoniifolius, C. esculenta, and X. sagittifolium indicates the putative protease cleavage site Q/A. The DAG motif is indicated by $\Delta$ and the repetitive amino acid is indicated by $\rightleftarrows$. The proline residues are indicated by $\leftrightharpoons$. The potential glycosylation motifs are underlined with bold lines $\_$and the conserved motifs (ADDTV, DEVVL, MVWCIDN, QMKAA) are indicated by 
Table 1. Sequence identity matrix showing the relative nucleotide identity of the three DsMV isolate under study with other DsMV isolates

\begin{tabular}{|c|c|c|c|c|c|c|c|c|c|c|c|c|c|c|c|c|c|c|c|c|c|c|}
\hline$\stackrel{\hat{1}}{\mathscr{J}}$ & 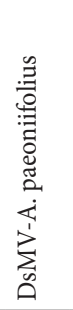 & $\sum_{\substack{\infty \\
0}}^{\stackrel{0}{*}}$ & 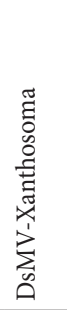 & 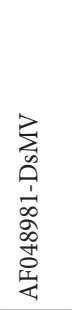 & 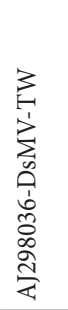 & 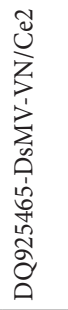 & 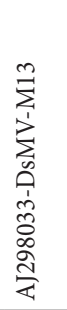 & 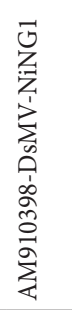 & 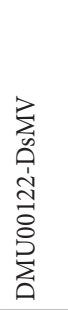 & 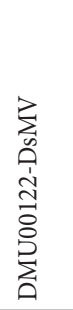 & 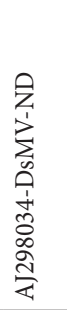 & 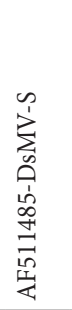 & 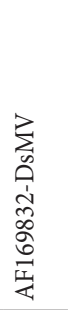 & 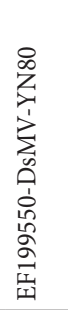 & 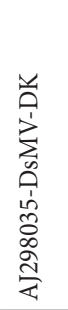 & 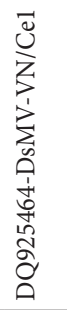 & 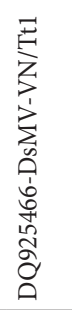 & 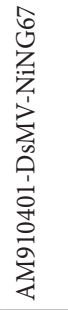 & 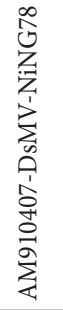 & 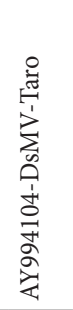 & 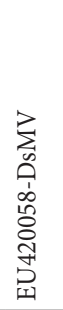 & 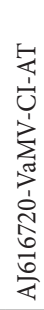 \\
\hline DsMV-A. paeoniifolius & ID & & & & & & & & & & & & & & & & & & & & & \\
\hline DsMV-Taro & 0.927 & ID & & & & & & & & & & & & & & & & & & & & \\
\hline DsMV-Xanthosoma & 0.75 & 0.728 & ID & & & & & & & & & & & & & & & & & & & \\
\hline AF048981-DsMV & 0.906 & 0.877 & 0.739 & ID & & & & & & & & & & & & & & & & & & \\
\hline AJ298036-DsMV-TW & 0.877 & 0.854 & 0.725 & 0.871 & ID & & & & & & & & & & & & & & & & & \\
\hline DQ925465-DsMV-VN/Ce2 & 0.862 & 0.836 & 0.72 & 0.865 & 0.904 & ID & & & & & & & & & & & & & & & & \\
\hline AJ298033-DsMV-M13 & 0.869 & 0.841 & 0.72 & 0.881 & 0.891 & 0.88 & ID & & & & & & & & & & & & & & & \\
\hline AM910398-DsMV-NiNG1 & 0.796 & 0.77 & 0.67 & 0.798 & 0.818 & 0.822 & 0.81 & ID & & & & & & & & & & & & & & \\
\hline DMU00122-DsMV & 0.809 & 0.786 & 0.675 & 0.813 & 0.83 & 0.832 & 0.827 & 0.962 & ID & & & & & & & & & & & & & \\
\hline DMU08124-DsMV & 0.862 & 0.839 & 0.712 & 0.878 & 0.899 & 0.861 & 0.894 & 0.809 & 0.82 & ID & & & & & & & & & & & & \\
\hline AJ298034-DsMV-ND & 0.867 & 0.834 & 0.715 & 0.869 & 0.884 & 0.861 & 0.907 & 0.819 & 0.823 & 0.891 & ID & & & & & & & & & & & \\
\hline AF511485-DsMV-S & 0.872 & 0.842 & 0.721 & 0.872 & 0.883 & 0.871 & 0.914 & 0.808 & 0.827 & 0.901 & 0.886 & ID & & & & & & & & & & \\
\hline AF169832-DsMV & 0.819 & 0.791 & 0.678 & 0.821 & 0.844 & 0.816 & 0.834 & 0.818 & 0.836 & 0.84 & 0.819 & 0.841 & ID & & & & & & & & & \\
\hline EF199550-DsMV-YN80 & 0.792 & 0.774 & 0.662 & 0.793 & 0.818 & 0.828 & 0.809 & 0.913 & 0.925 & 0.811 & 0.804 & 0.808 & 0.826 & ID & & & & & & & & \\
\hline AJ298035-DsMV-DK & 0.819 & 0.794 & 0.693 & 0.812 & 0.825 & 0.833 & 0.82 & 0.83 & 0.839 & 0.816 & 0.813 & 0.811 & 0.814 & 0.861 & ID & & & & & & & \\
\hline DQ925464-DsMV-VN/Cel & 0.785 & 0.765 & 0.678 & 0.79 & 0.793 & 0.77 & 0.785 & 0.779 & 0.792 & 0.787 & 0.775 & 0.787 & 0.797 & 0.8 & 0.763 & ID & & & & & & \\
\hline DQ925466-DsMV-VN/Tt1 & 0.737 & 0.73 & 0.627 & 0.734 & 0.732 & 0.72 & 0.738 & 0.715 & 0.697 & 0.726 & 0.719 & 0.721 & 0.696 & 0.704 & 0.724 & 0.672 & ID & & & & & \\
\hline AM910401-DsMV-NiNG67 & 0.794 & 0.774 & 0.673 & 0.801 & 0.817 & 0.828 & 0.813 & 0.973 & 0.944 & 0.808 & 0.808 & 0.808 & 0.819 & 0.917 & 0.839 & 0.792 & 0.716 & ID & & & & \\
\hline AM910407-DsMV-NiNG78 & 0.77 & 0.75 & 0.65 & 0.78 & 0.78 & 0.79 & 0.78 & 0.9 & 0.88 & 0.79 & 0.77 & 0.79 & 0.81 & 0.9 & 0.85 & 0.8 & 0.71 & 0.91 & ID & & & \\
\hline AY994104-DsMV-Taro & 0.701 & 0.688 & 0.623 & 0.692 & 0.697 & 0.697 & 0.689 & 0.717 & 0.713 & 0.694 & 0.691 & 0.693 & 0.715 & 0.725 & 0.72 & 0.71 & 0.646 & 0.718 & 0.72 & ID & & \\
\hline EU420058-DsMV & 0.683 & 0.669 & 0.8 & 0.693 & 0.686 & 0.682 & 0.676 & 0.675 & 0.664 & 0.681 & 0.681 & 0.677 & 0.645 & 0.662 & 0.68 & 0.645 & 0.774 & 0.671 & 0.67 & 0.616 & ID & \\
\hline AJ616720-VaMV-CI-AT & 0.67 & 0.66 & 0.6 & 0.67 & 0.69 & 0.68 & 0.68 & 0.69 & 0.68 & 0.67 & 0.67 & 0.68 & 0.68 & 0.7 & 0.71 & 0.67 & 0.75 & 0.7 & 0.7 & 0.71 & 0.72 & ID \\
\hline
\end{tabular}

DTG sequence in DsMV-Xan1 instead of the aphid transmission motif DAG, as reported in other potyviruses (Atreya et al., 1991; Pappu et al., 1994). In our aphid transmission experiments using Aphis craccivora and Myzus persicae we could not possibly transmit the virus to any of the three plants. The loss of aphid transmission could be attributed to the single amino acid substitutions in the DAG motif. Moreover we couldn't find any aphids species feeding naturally on the plants in the field. Even though Aphis craccivora and Myzus persicae are the common aphids for the transmission of the DsMV viruses, further analysis of our isolates using other aphid's species needs to be carried out. Following the aphid transmission motif an unusual and unique stretch of 36 amino acids, which is repetitive and rich in threonine and asparagine is found in all the isolates, followed by several proline residue repeats. The $\mathrm{CP}$ of all the three isolates has several threonine and asparagine (NTT, NGT, NNT, NVT NGTS, NVST, and NVS) rich potential N-glycosylation motifs in the central region, including some that cluster among the repeat sequences. Similar motifs were also found in the CP of other DsMV isolates (Pappu et al., 1994). Analysis of the po- tyviral conserved core CP MVWCIEN motif region shows that, a single base substitution occurs with $\mathrm{E} / \mathrm{Y}$ in DsMV-Xan1 and with E/D in DsMV-Amp1 and DsMV-Ta1, while the potyviral QMKAA motif was conserved in DsMV-Xan1 and a single base substitution of K/M in DsMV-Amp1 and DsMV-Ta1.

Among the edible aroids of worldwide economic importance, A. paeoniifolius, C. esculenta, and X. sagittifolium play a very important role in the socio-economical and agricultural scenario of India. Since the DsMV infection on the tuber crops results in reduction in the tuber yield, thus becoming major constraints for the farmer, an effective management practice for the virus needs to be developed. As a first step towards development of virus resistant varieties, the molecular characterization of the virus has to be carried out. Moreover there is limited sequence information of DsMV infecting these major edible aroids from India. Hence a comparative analysis of the DsMV viral isolates at the nucleic acid level would facilitate the development of methods for the specific diagnosis, their pattern of infection and ultimately for the development of virus resistant varieties. 
Acknowledgements. The authors wish to thank the Indian Council of Agricultural Research, New Delhi for funding under "ICAR Network Project on Diagnostics of Emerging Plant Viruses" and the Director, Central Tuber Crops Research Institute, Thiruvananthapuram for the facilities and support. The authors would like to thank Dr. Mathews L. Paret (Assistant professor, NFREC, University of Florida) for his assistance with review of manuscript.

\section{References}

Adams MJ, Antoniw JF, Fauquet CM (2005): Molecular criteria for genus and species discrimination within the family Potyviridae. Arch. Virol. 150, 459-479. http://dx.doi. org/10.1007/s00705-004-0440-6

Atreya PL, Atreya CD, Pirone TP (1991): Amino acid substitutions in the coat protein result in the loss of insect transmissibility of a plant virus. Proc. Natl. Acad. Sci. USA 88, 7887-7891. http://dx.doi.org/10.1073/pnas.88.17.7887

Bateson MF, Lines RE, Revill P, Chaleeprom W, Ha CV, Gibbs AJ, Dale JL (2002): On the evolution and molecular epidemiology of the potyvirus Papaya ringspot virus. J. Gen. Virol. 83, 2575-2585.

Binoy B, Hegde V, Makeshkumar T, Jeeva ML (2010): Rapid detection and identification of potyvirus infecting Colocasia esculenta (L.) Schott by reverse transcription-polymerase chain reaction. J. Root Crops 36, 88-94.

Brunt AA, Crabtree K, Dallwitz MJ, Gibbs AJ, Watson L (1996): Viruses of Plants. CAB International, Wallingford, UK, pp. 1484.

Chen J, Chen J, Chen J, Adams MJ (2001): Molecular characterization of an isolate of Dasheen mosaic virus from Zantedeschia aethiopica in China and comparisons in the genus Potyvirus. Arch. Virol. 146, 1821-1829. http:// dx.doi.org/10.1007/s007050170068

Devereux J, Haeberli P, Smithies O (1984): A comprehensive set of sequence analysis programs for the VAX. Nucleic Acids Res. 12, 387-395. http://dx.doi.org/10.1093/ $\underline{\text { nar/12.1Part1.387 }}$

Farreyrol K, Pearson MN, Grisoni M, Cohen D, Beck D (2006): Vanilla mosaic virus isolates from French Polynesia and the Cook Islands are Dasheen mosaic virus strains that exclusively infect vanilla. Arch. Virol. 151, 905-919. http://dx.doi.org/10.1007/s00705-005-0680-0

Garcia-Arenal F, Fraile A, Malpica JM (2001): Variability and genetic structure of plant virus populations. Ann. Rev. Phytopathology 39, 157-186. http://dx.doi.org/10.1146/ annurev.phyto.39.1.157

Hall TA (1999): Bioedit: A user-friendly biologically sequence alignment editor and analysis program for windows 95/98/NT. Nucleic Acids Symposium series 41, 95-98.

Jennings DL (1987): Starch crops. In Christine BR (Eds): CRC Handbook of Plant Science in Agriculture. Vol. II. CRC Press, Inc. Boca Raton, Florida, USA, pp. 137-143.

Malathi VG, Shanta P (1981): A transmissible mosaic disease of edible aroids. J. Root Crops 7, 77-78.
Martin D, Rybicki E (2000): RDP: detection of recombination amongst aligned sequences. Bioinformatics 16, 562-563. http://dx.doi.org/10.1093/bioinformatics/16.6.562

Misra RS, Nedunchezhiyan M, Swamy TMS, Edison S (2002): Mass multiplication technique for producing quality planting material of Amorphophallus paeoniifolius. Tropical Sci. 34, 371-376.

Ndoumou DO, Tsala GN, Kanmegne G, Balangé AP (1995): In vitro induction of multiple shoots, plant generation and tuberization from shoot tips of cocoyam. C. R. Acd. Sci. Paris, Sciences de la vie/Life Sciences 318, 773-778.

NehalKhan M, Aswathi LP, Singh PK (2006): Survey on symptomatology and assessment of yield losses due to viral diseases in Elephant foot yam (Amorphophallus paeoniifolius Blume). J. Root Crops 32, 90-93.

Nyochembeng L, Garton S (1998): Plant regeneration from cocoyam callus derived from shoot tips and petioles. Plant Cell, Tissue and Organ Culture 53, 127-134. http://dx.doi. org/10.1023/A:1006084207636

Onwueme IC, Charles WB (1994): Cultivation of cocoyam. In Tropical root and tuber crops. Production, perspectives and future prospects. FAO Plant Production and Protection Paper 126, Rome, pp. 139-161.

Pandit MK, Mukhopadhyay S, Devonshire BJ, Jones P (2001): First report of Dasheen mosaic virus in Elephant foot yam in India. Plant Pathol. 50, 802. http://dx.doi.org/10.1046/ j.1365-3059.2001.00625.x

Pappu SS, Pappu HR, Rybicki EP, Niblett CL (1994): Unusual amino terminal sequence repeat characterizes the capsid protein of dasheen mosaic potyvirus. J. Gen. Virol. 75, 239-242. http://dx.doi.org/10.1099/0022-1317-75-1-239

Reyes G, Ramsell JNE, Nyman M, Kvarnheden A (2009): Sequence characterisation of Dasheen mosaic virus isolates from cocoyam in Nicaragua. Arch. Virol. 154, 159-162. http:// dx.doi.org/10.1007/s00705-008-0257-9

Sambrook J, Russel DW (2001): Molecular Cloning: A laboratory Manual. Third edition, Cold Spring Harbour Laboratory Press, USA.

Srinivas T, Ramanathan S (2005): A study on economic analysis of Elephant foot yam production in India. Agri. Econ. Res. Rev. 18, 241-252.

Tambong JT, Ndzana X, Wutoh JG, Dadson R (1997): Variability and germplasm loss in the Cameroon national collection of cocoyam (Xanthosoma sagittifolium Schott (L.)). Plant Genetic Resources Newletters 112, 49-54.

Tajima F, Nei M (1984): Estimation of evolutionary distance between nucleotide sequences. Mol. Bio. Evol. 1, 269-285.

Thompson JD, Higgine DG, Gibson TJ (1994): CLUSTAL W: Improving the sensitivity of multiple sequence alignment through sequence weighting, position specific gap penalties and weight matrix choice. Nucleic Acids Res. 22, 4673-4680. http://dx.doi.org/10.1093/nar/22.22.4673

Van de Peer Y, De Wachter Y (1994): TREECON for Windows: a software package for the construction and drawing of evolutionary trees for the Microsoft Windows environment. Computational Applications in Bioscience 10, 569-570.

Yu MH, Frenkel MJ, Mckern NM, Shukla DD, Strike P, Ward CW (1989): Coat protein of potyviruses 6. Amino acid 
sequences suggest watermelon mosaic virus 2 and soybean mosaic virus- $\mathrm{N}$ are strains of the same potyvirus. Arch. Virol. 105, 55-64 http://dx.doi.org/10.1007/ BF01311116

Zettler FW, Abo El-Nil MM, Hartman RD (1978): Dasheen mosaic virus. CMI/AAB Descriptions of Plant Viruses, No. 191.

Zettler FW, Hartman RD (1986): Dasheen mosaic virus and its control in cultivated aroids. Plant virus diseases of horticultural crops in the tropics and subtropics. In Food and Fertilizer Technology Center Book Series No. 33, pp. 91-100.
Zettler FW, Hartman RD (1987): Dasheen mosaic virus as a pathogen of cultivated aroids and control of the virus by tissue culture. Plant Dis. 71, 958-963. http://dx.doi. org/10.1094/PD-71-0958

Zettler FW, Jackson GVH, Frison EA (1989): Technical Guidelines for the Safe Movement of Edible Aroid Germplasm. FAO/ IBPGR (Ed.): Food and Agriculture Organization of the United Nations, Rome/International Board for Plant Genetic Resources, Rome. 